\title{
Early detection of impending slope failure in open pit mines using spatial and temporal analysis of real aperture radar measurements
}

\author{
G.J. Dick Department of Earth and Ocean Sciences, University of British Columbia, Canada \\ E. Eberhardt Department of Earth and Ocean Sciences, University of British Columbia, Canada \\ D. Stead Department of Earth Sciences, Simon Fraser University, Canada
}

N.D. Rose Piteau Associates Engineering Ltd., Canada

\begin{abstract}
Slope monitoring in open pit mines is an essential component of day-to-day operations and plays a key role in assisting geotechnical engineers and mine operators in maintaining mine safety and production schedules. Pit slope monitoring techniques have advanced significantly within the past decade, most notably in ground-based radar technology. Ground-based radar allows real-time monitoring of slope deformation across a broad coverage area, alerting mine staff to wall movements exceeding established thresholds. Line-of-sight measurements derived from the radar can be presented as 3D point clouds for the scan area, allowing mine staff to view the distribution of slope movements across the pit wall with each progressive scan.

This paper presents a new methodology for spatial and temporal analysis of deformation point clouds captured by ground-based radar. The methodology builds on two existing early warning methods, the Fukuzono inverse-velocity method and the SLOpe gradient (SLO) method, which are based on the analysis of point measurement data derived from traditional geodetic prism monitoring. However, similar methodologies that fully utilise the spatial and temporal characteristics of ground-based radar data are yet to be developed.

Radar data from historical slope failures captured by GroundProbe Slope Stability Radar (SSR) at a number of hard rock mines was utilised in the development of the new spatial and temporal analysis methodology. A slope failure that occurred at an open pit copper mine is presented throughout as a case example. The spatial analysis component of the methodology uses a benchmark point (or pixel), based on an alarm threshold specific to each failure case, and averaged deformation increments based on a percentage of the deformation measured by the benchmark pixel at the time of alarm. The temporal analysis component of the methodology examines deformation and velocity trends for all spatial analysis cases. The results of the spatial and temporal analysis were then used to evaluate the inverse-velocity and SLO time of failure prediction methods. Overall, the proposed methodology will improve, and provide a more systematic means of interpreting spatial and temporal ground-based radar data, aiding geotechnical engineers in managing slope movement alarms and alarm responses to provide a safer working environment for mine employees.
\end{abstract}

\section{Introduction}

The use of ground-based radar for monitoring pit slope wall instabilities has become the state of practice in many of today's open pit mine operations. Ground-based radar systems allow real-time monitoring of active slope instabilities, providing 3D slope deformation point clouds derived from line-of-sight measurements. These 3D point clouds allow mine staff to view the distribution of slope movements across the pit wall with each progressive scan.

However, conventional treatments of pit wall monitoring data for early warning have been developed based on point measurement deformation data, as for example derived from geodetic monitoring. Periodic 
measurement of deformation over time are recorded for a series of survey prisms across a slope, which are then analysed for accelerations and extrapolated in order to predict impending failure. Examples include the inverse-velocity method (Fukuzono, 1985) and the SLOpe gradient (SLO) method (Mufundirwa et al., 2010). Using point measurements, prism monitoring is susceptible to uncertainty relating to the geological conditions and slope kinematics controlling the instability mechanism. The detection of accelerating behaviour may either be an early warning of impending failure or a false alarm related to highly localised movements in the immediate vicinity of the prism. Similarly, the interpolation of slope behaviour between prism locations may result in deformation influenced by large scale geological structures being misinterpreted or missed altogether; ground-based radar overcomes these limitations.

This paper presents a new methodology for spatial and temporal analysis of deformation point clouds captured by ground-based radar. The use of the inverse-velocity method (Fukuzono, 1985) and the SLO method (Mufundirwa et al., 2010) to predict the time of failure (TOF) was evaluated and extended to radar data. A slope failure at an open pit copper mine, captured by GroundProbe Slope Stability Radar (SSR), is used to demonstrate how the new spatial and temporal analysis method is applied. This failure involved a one million tonne planar failure occurring in metasedimentary rocks.

\section{Background}

\subsection{TOF forecast methods}

Two TOF prediction methods were assessed: the inverse-velocity method (Fukuzono, 1985), and the SLO gradient method (Mufundirwa et al., 2010). Both methods utilise non-rigorous, linear regression trends using time-deformation rate (velocity) measurements.

\subsubsection{Inverse-velocity method (Fukuzono, 1985)}

Fukuzono (1985) found that the time to accelerating creep failure under gravity loading was inversely proportional to the deformation rate (velocity). By plotting the inverse-velocity versus time curve, one can estimate the time of failure by extrapolating the curve to the $x$-axis (inverse-velocity $=0$ ). Extrapolating inverse-velocity to the $x$-axis is much easier than extrapolating a hyperbolic curve to a vertical asymptote when using the deformation rate. Fukuzono (1985) presented three types of inverse-velocity curves based on laboratory results simulating rain-induced landslides in soil (shown in Figure 1). The three curves shown in Figure 1 are defined by the following equation:

$$
V^{-1}=[A(\alpha-1)]^{\frac{1}{\alpha-1}}\left(t_{f}-t\right)^{\frac{1}{1-\alpha}}
$$

Where $V^{-1}$ is the inverse of the deformation rate, $t$ is time, $A$ and $\alpha$ are constants, and $t_{f}$ is the time of failure. Based on the laboratory results, Fukuzono (1985) found that $\alpha$ ranged between 1.5 and 2.2; $\alpha=2$ represents a linear inverse-velocity trend. $A$ is the slope of the inverse-velocity trend, which is specific to each data set.

Rose and Hungr (2007) successfully predicted the time of slope failure in three hard rock open pit mines that occurred between 2001 and 2005 and observed that the inverse-velocity plot often approaches linearity, particularly close to the time of failure. Their work recommends the use of linear fits updated on an ongoing basis and any best fit functions be revised using the latest data. This assumption of linearity has been shown to be reasonable by many authors for accelerating creep occurring under constant effective stress. Rose and Hungr (2007) advise that the inverse-velocity method should not be used for rock slides dominated by brittle failure, where little time may precede a potential failure event, and care should be taken when dealing with smaller failures in strong rock. 


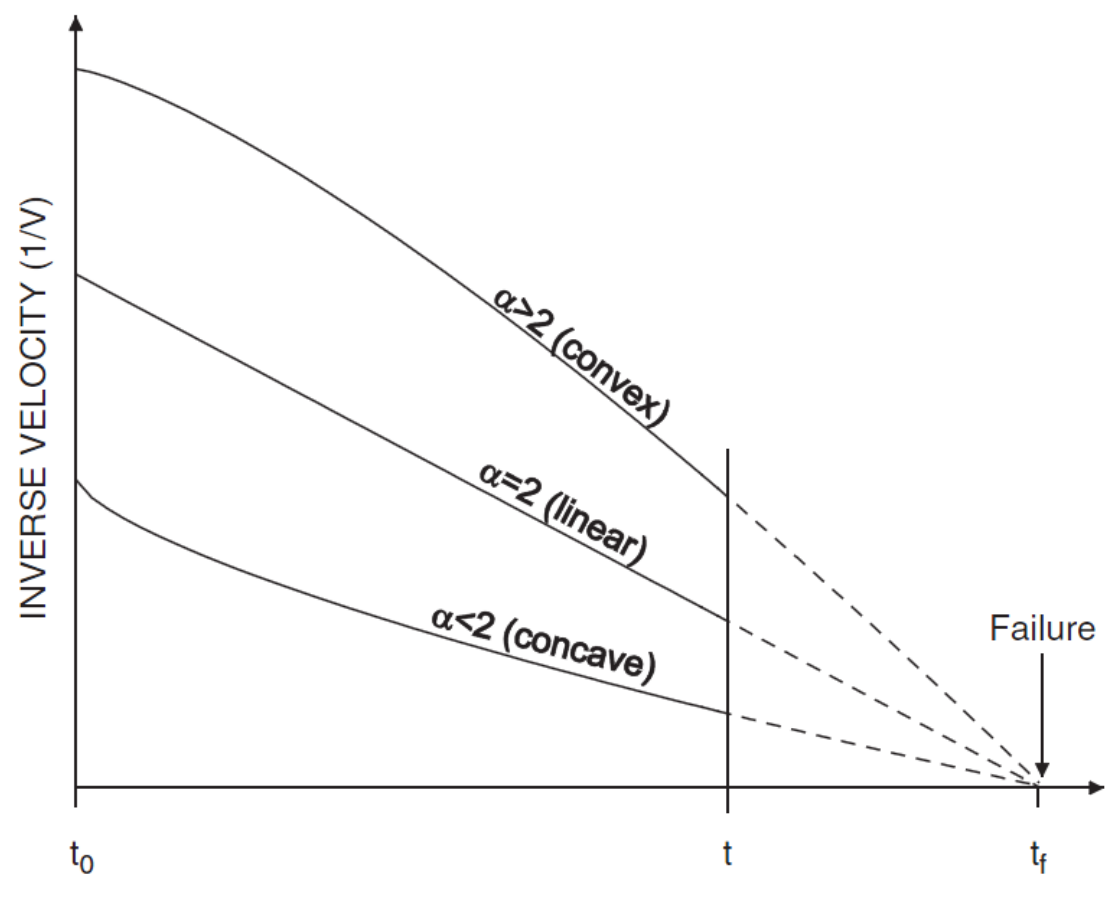

TIME

Figure 1 Inverse-velocity versus time of displacement preceding failure (after Fukuzono, 1985)

\subsubsection{SLO method (Mufundirwa et al., 2010)}

Mufundirwa et al. (2010) developed a method to predict the time of geomechanical failure based on the slope (gradient) of the $t(d u / d t)-d u / d t$ curve, termed SLO. Where $t$ is time and $d u / d t$ is the deformation rate. Mufundirwa et al. (2010) derived this relationship based on the strain divergence in the terminal phase of creep failure in rocks proposed by Fukui and Okubo (1997), resulting in the following relationship (please see Mufundirwa et al. (2010) for full derivation):

$$
t \frac{d u}{d t}=T_{f} \frac{d u}{d t}-B
$$

Where $t$ is time, $T_{f}$ is the failure time, $d u / d t$ is the deformation rate, and $B$ is a constant. From Equation 2, the time of failure $\left(T_{f}\right)$ can be evaluated as the slope of the $t(d u / d t)-d u / d t$ curve. Figure 2 shows how the SLO method is applied to deformation rate measurements from a GroundProbe SSR.
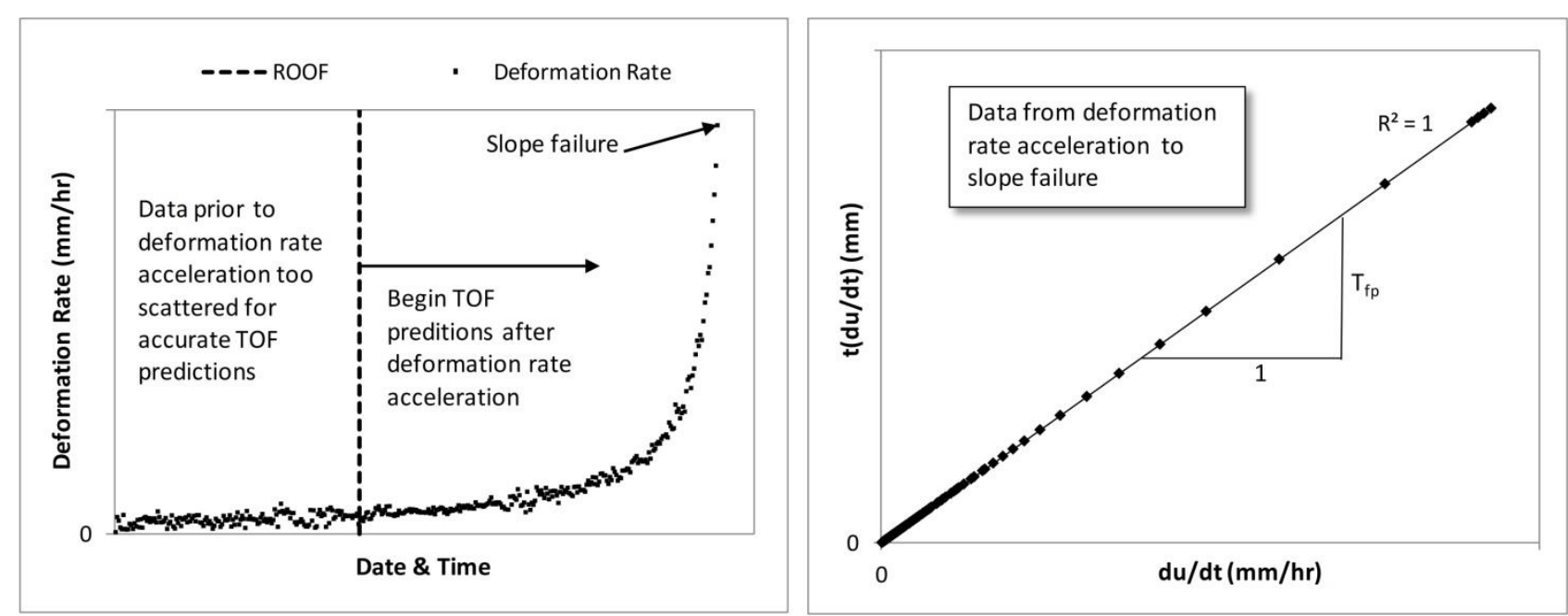

Figure 2 Example deformation rate curve and its corresponding $\mathrm{t}(\mathrm{du} / \mathrm{dt})$-du/dt plot illustrating the SLO TOF prediction method 


\subsection{GroundProbe SSR measurements}

Radar data from historical slope failures captured by GroundProbe SSR at a number of hard rock mines was utilised in the development of the spatial and temporal analysis method presented here. The SSR uses real-aperture radar on a stationary platform to monitor slope deformation remotely, without the need for reflectors on the slope face (Reeves et al., 2001). Remote monitoring using the SSR allows for active monitoring of a slope with deformation alerts of sub-millimetre precision, making the data available for interpretation usually within minutes (Harries et al., 2006).

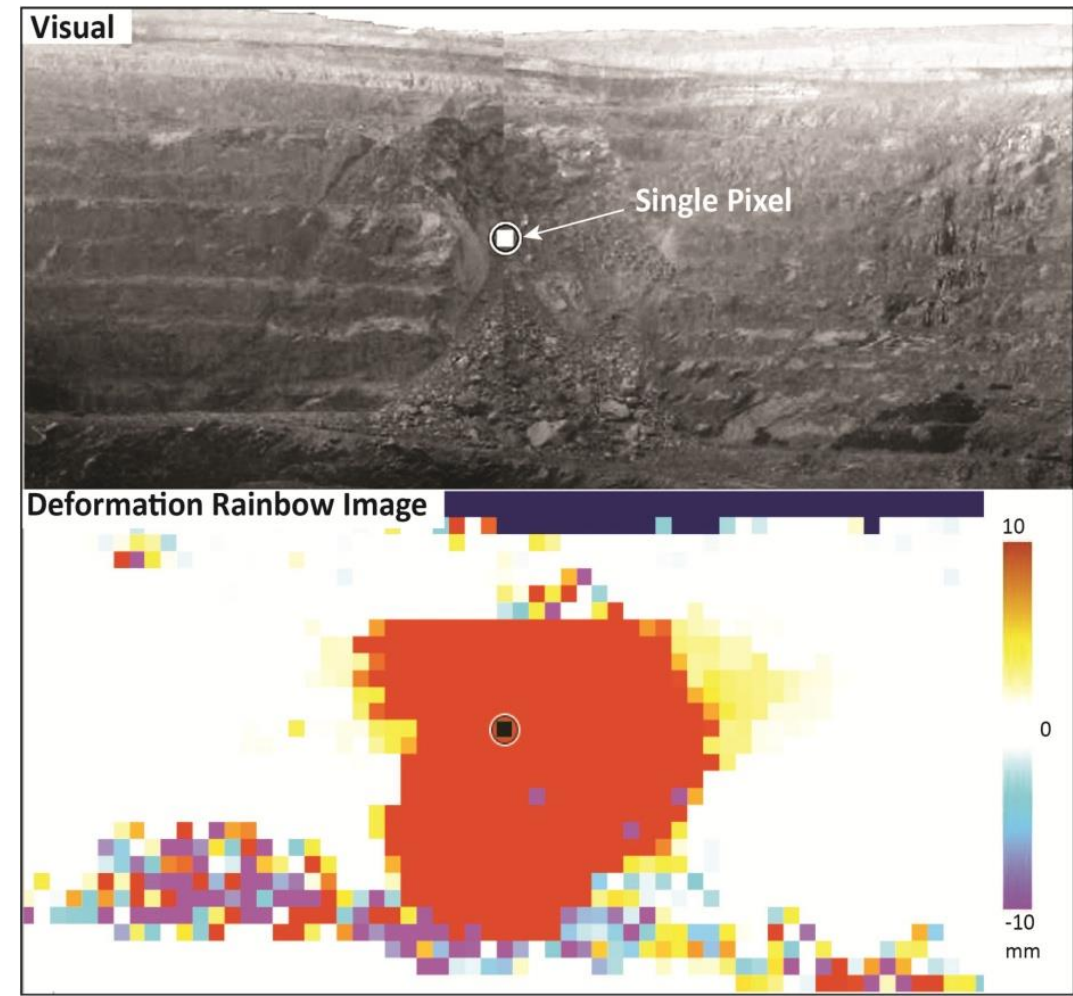

\section{Single Pixel Measurements}

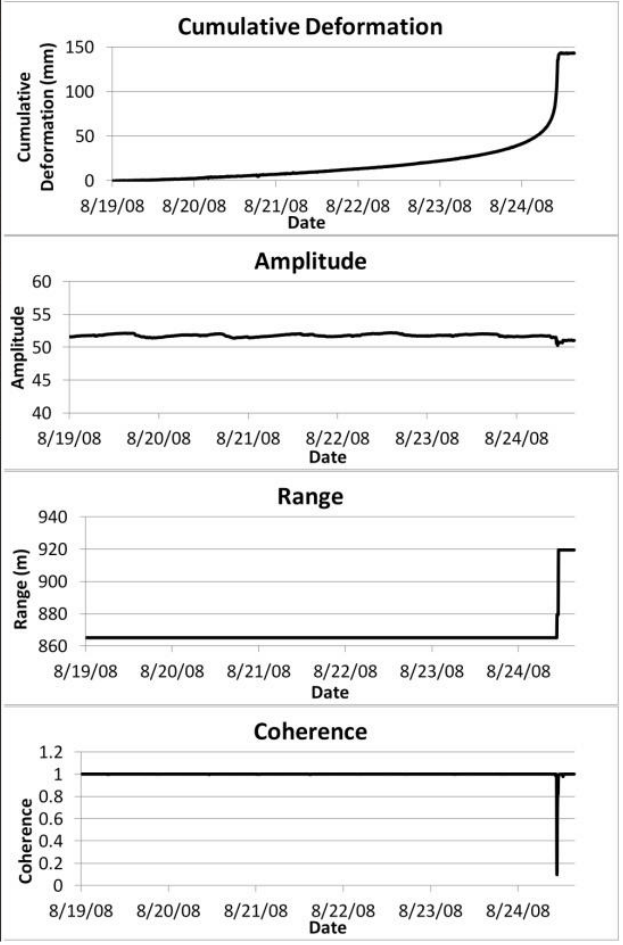

Figure 3 Copper mine slope failure measurements captured by GroundProbe SSR

The deformation, amplitude, range, and coherence measurements from the SSR were analysed spatially and temporally. Figure 3 shows the measurements of a single pixel from the copper mine slope failure captured by a SSR. The deformation plot provides the cumulative slope displacement (between the SSR and the slope) with each progressive scan from the beginning of deployment. The amplitude is the signal strength of the reflection of the slope material. The range is the distance between the SSR dish and the slope. The coherence is a correlation measurement based on the range and amplitude between the current scan and the immediately preceding scan, with values close to 1.0 indicating little difference in range and amplitude between the two scans. The coherence measurement is especially useful for establishing the time of slope collapse. The coherence plot in Figure 3 shows a downward spike to a coherence of approximately 0.1 , indicating a large difference in range and amplitude between the two scans. This downward spike in coherence coincides closely with the peak of the deformation plot, indicative of impending slope failure. Figure 4 shows the coherence image of each pixel in the scan area for the SSR scan immediately before and after the slope failure occurring at the copper mine.

The dimensions (or size) of a pixel depends on how far the SSR is placed from the slope, with higher pixel resolution (smaller pixels) being achieved the closer the SSR is setup to the slope. When more than one pixel is selected, the deformation for the area of pixels is based on an amplitude-weighted average; this gives priority to the deformation of pixels that have a stronger return signal (GroundProbe Pty Ltd, 2012). 

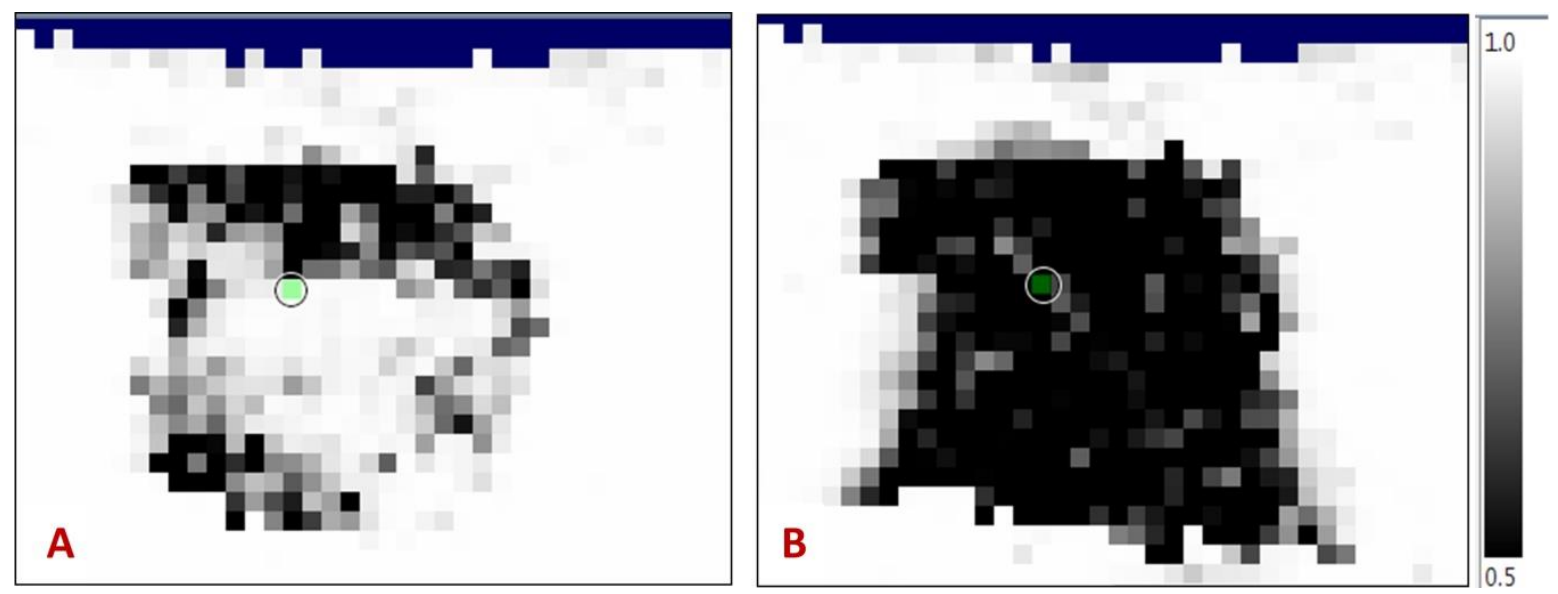

Figure 4 Coherence image of copper mine slope failure; (a) scan immediately prior to slope collapse; (b) scan immediately after slope collapse

\subsection{Unstable slope terminology}

Many authors use different nomenclature interchangeably when discussing slope instabilities. For example, the term 'failure' has been applied loosely in literature to describe almost every degree of instability and type of behaviour possible (Mercer, 2006). For the purpose of this paper, the definitions presented in Table 1 will be used. The definitions of the terminology pertain mainly to engineered slopes in open pit mines in relation to time-deformation trends. The terminology has been chosen to be consistent with those commonly used by geotechnical engineers and for which they are best understood. A new term, the revised onset-of-failure (ROOF), is being defined for the purpose of improving the accuracy of the TOF predictions.

\section{Table 1 Nomenclature for unstable slopes used in this paper}

\begin{tabular}{|c|c|}
\hline Term & Definition \\
\hline Instability & $\begin{array}{l}\text { Deformational movement or behaviour that does not involve collapse or } \\
\text { failure (Mercer, 2006) }\end{array}$ \\
\hline Progressive deformation & $\begin{array}{l}\text { Accelerating behaviour leading eventually to slope collapse (Zavodni and } \\
\text { Broadbent, 1980) }\end{array}$ \\
\hline Regressive deformation & $\begin{array}{l}\text { Decelerating behaviour leading eventually to a stable slope (Zavodni and } \\
\text { Broadbent, 1980) }\end{array}$ \\
\hline Onset-of-failure (OOF) & $\begin{array}{l}\text { Point defining the transition from a regressive state to a progressive stage } \\
\text { ultimately leading to slope collapse (Mercer, 2006; Zavodni and Broadbent, } \\
\text { 1980) }\end{array}$ \\
\hline $\begin{array}{l}\text { Revised onset-of- failure } \\
\text { (ROOF) }\end{array}$ & $\begin{array}{l}\text { Point during the progressive deformation stage where the time-deformation } \\
\text { measurements show a considerable decrease in noise, marking the time to } \\
\text { begin TOF predications }\end{array}$ \\
\hline Slope failure & Theoretical, taken at the end of the progressive deformation stage \\
\hline Slope collapse & $\begin{array}{l}\text { Physical, complete overall loss of rock mass integrity and structure (Mercer, } \\
\text { 2006) }\end{array}$ \\
\hline $\begin{array}{l}\text { Predicted life } \\
\text { expectancy }\end{array}$ & $\begin{array}{l}\text { Difference between the predicted time of failure and the time the prediction } \\
\text { is made (Mufundirwa et al., 2010) }\end{array}$ \\
\hline
\end{tabular}

Figure 5 illustrates how the onset-of-failure (OOF), revised onset-of-failure (ROOF), and slope failure times were estimated for the spatial and temporal analysis based on the time versus deformation, deformation rate (or velocity), and inverse-velocity plots. The OOF point was estimated using the cumulative 
deformation versus time plots where an increase in deformation rate was observed leading into a progressive deformation stage and when the inverse-velocity trend began showing a consistent linear trend towards the $x$-axis. The ROOF marks the point during the progressive stage where a considerable decrease in data noise is observed, primarily based on the inverse-velocity trend, and the start point for TOF predictions. The slope failure point was defined as the end of the progressive deformation stage, typically coinciding with maximum observed deformation rate followed by a drastic decrease. The slope collapse time was determined based on the coherence image output from the SSR taken as the time of the scan prior to collapse, and validated with photographs and failure reports when available.

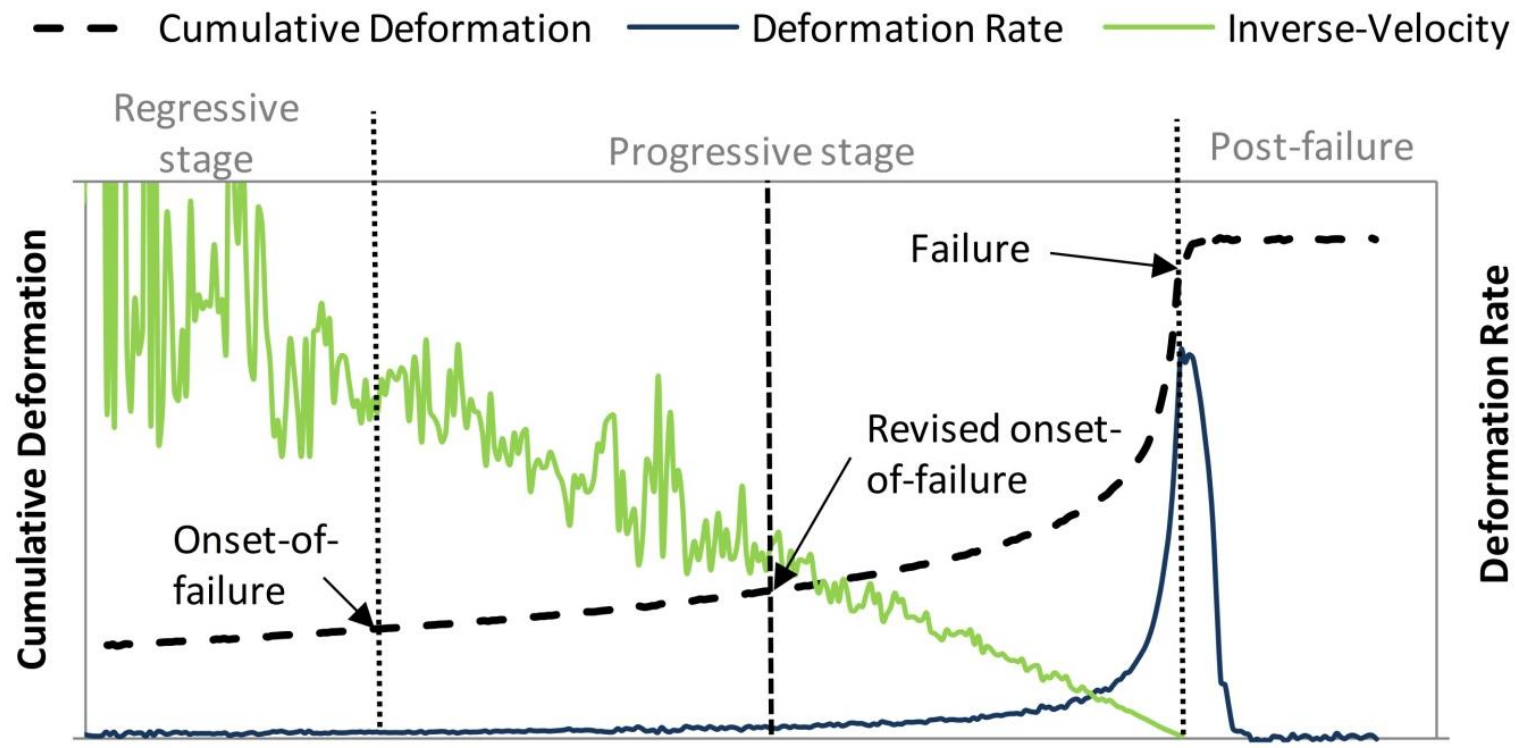

Time

Figure 5 Theoretical cumulative deformation and deformation rate versus time plots illustrating the time of onset-of-failure and slope failure

\section{Spatial and temporal analysis methodology}

The spatial and temporal analysis method presented here utilises the deformation point cloud recorded by a ground-based radar system, taking advantage of the aerial coverage rather than just individual pixels or prisms. Real-aperture radar data from historical slope failures captured by GroundProbe SSR at a number of hard rock mines was used to develop and test the methodology.

The spatial analysis component of the methodology uses a benchmark point (or pixel), based on an alarm threshold specific to each failure case. Additional surrounding pixels are then incrementally included based on a percentage of the deformation measured relative to the benchmark pixel at the time of alarm. Increments of 75,50 , and $25 \%$ of the first alarm benchmark pixel deformation or above will be used as examples to demonstrate the methodology. Only pixels adjacent to the benchmark pixel are to be included in the incremental selections. Figure 6 illustrates the pixels selected for analysis of the copper mine slope failure, where the outlined area and the pixels in green are selected for analysis. Note that some 'runaway pixels' (erroneous measurements due to sudden large changes in atmospheric conditions) were part of the data set and were not included in the selected pixels for analysis (blue pixels within outlined areas in Figure 6). The benchmark was selected as the first pixel to trigger a slope deformation alarm, which was set by the copper mine staff as $10 \mathrm{~mm}$ of cumulative deformation over four hours. The alarm pixel accrued $69.1 \mathrm{~mm}$ of cumulative deformation (since SSR deployment) at the time of the alarm, therefore the benchmark deformation in which the surrounding pixels were selected was $69.1 \mathrm{~mm}$. As a result, the pixels included in each incremental analysis accrued $51.8,34.6$, and $17.3 \mathrm{~mm}$ of cumulative deformation or above for the 75,50 , and $25 \%$ increments, respectively. 

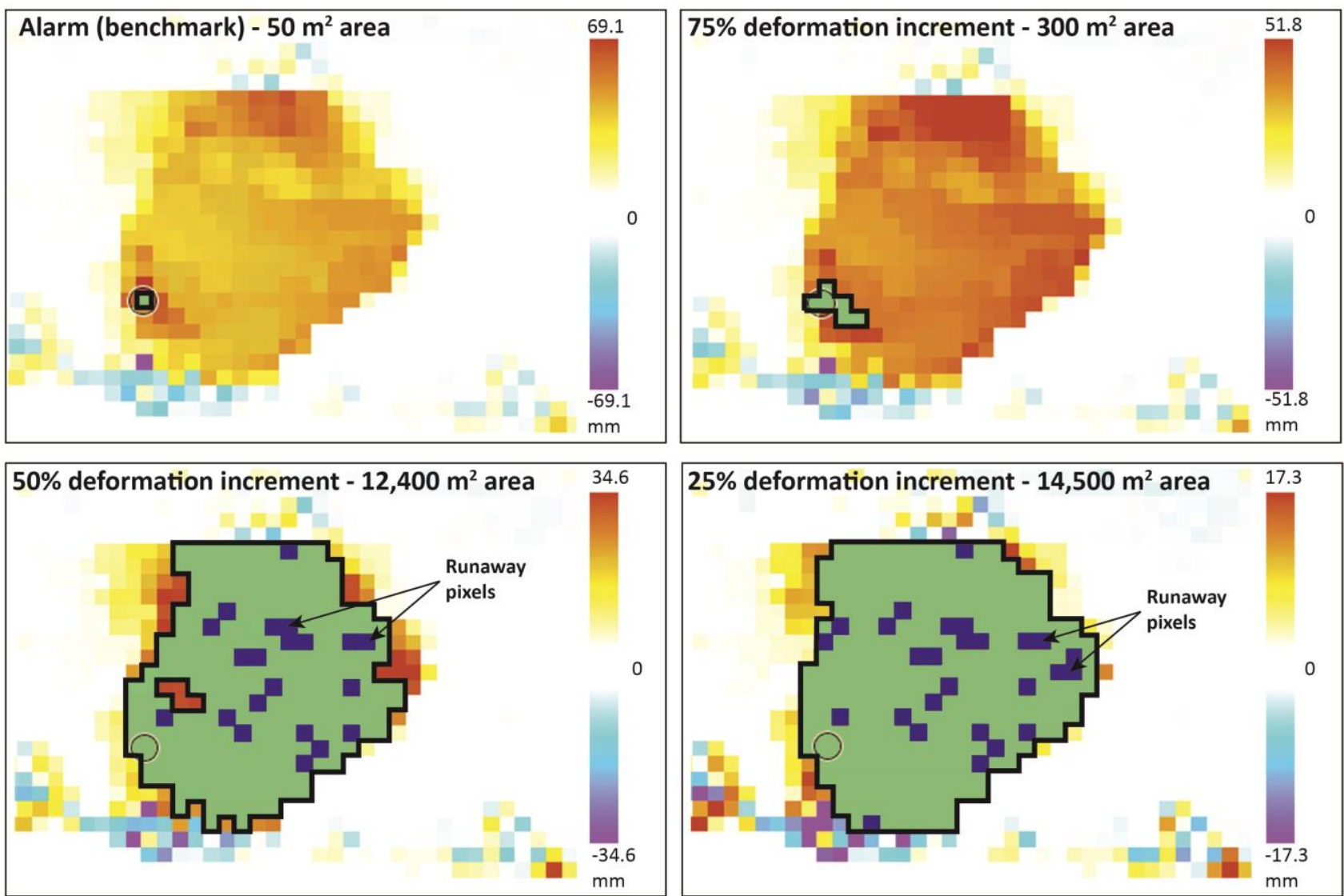

Figure 6 Deformation images illustrating the pixels selected for spatial and temporal analysis for the copper mine slope failure. Outlined pixels selected for analysis, 'runaway pixels' excluded from analysis

The temporal analysis component of the methodology examines deformation and deformation rate trends for all spatial analysis cases. The inverse-velocity and SLO methods can be applied to all deformation rate trends to evaluate the accuracy in predicting the time of slope failure. The SSR completes a slope scan approximately every 5 to 15 minutes, depending on the total scan area, which can result in a large amount of data noise; especially when looking at velocity trends. A data filtering method using every $n$th observation was used to calculate the deformation rate:

$$
v_{i}=\frac{d_{i}-d_{i-n}}{t_{i}-t_{i-n}}
$$

Where $v_{i}$ is the deformation rate, and $t_{i}$ and $d_{i}$ are the most recent time and deformation, respectively. Deformation rates were filtered over 1, 2 and 12 hours. Unfiltered data was also evaluated in the analysis $(n=1)$. Figure 7 shows the results of the spatial and temporal analysis method for the copper mine slope failure; the peak deformation rate is not shown in order to demonstrate the pre-failure trend.

Based on the definitions established in Section 2.3, the OOF, ROOF, and slope failure time can be different for each spatial analysis since the deformation versus time results differ in each case due to the averaging of multiple pixels. This difference is to be expected, especially for large areas, as rock mass behaviour will vary throughout the instability. The OOF and ROOF times can be equal for different deformation increments if the geotechnical engineer deems both definitions are met. The time of slope collapse remains the same in all cases since it is physically established based on coherence images, photos, and failure reports. From the SSR coherence images, the time of slope collapse is taken 25 August 2008 at 0214 hrs. 


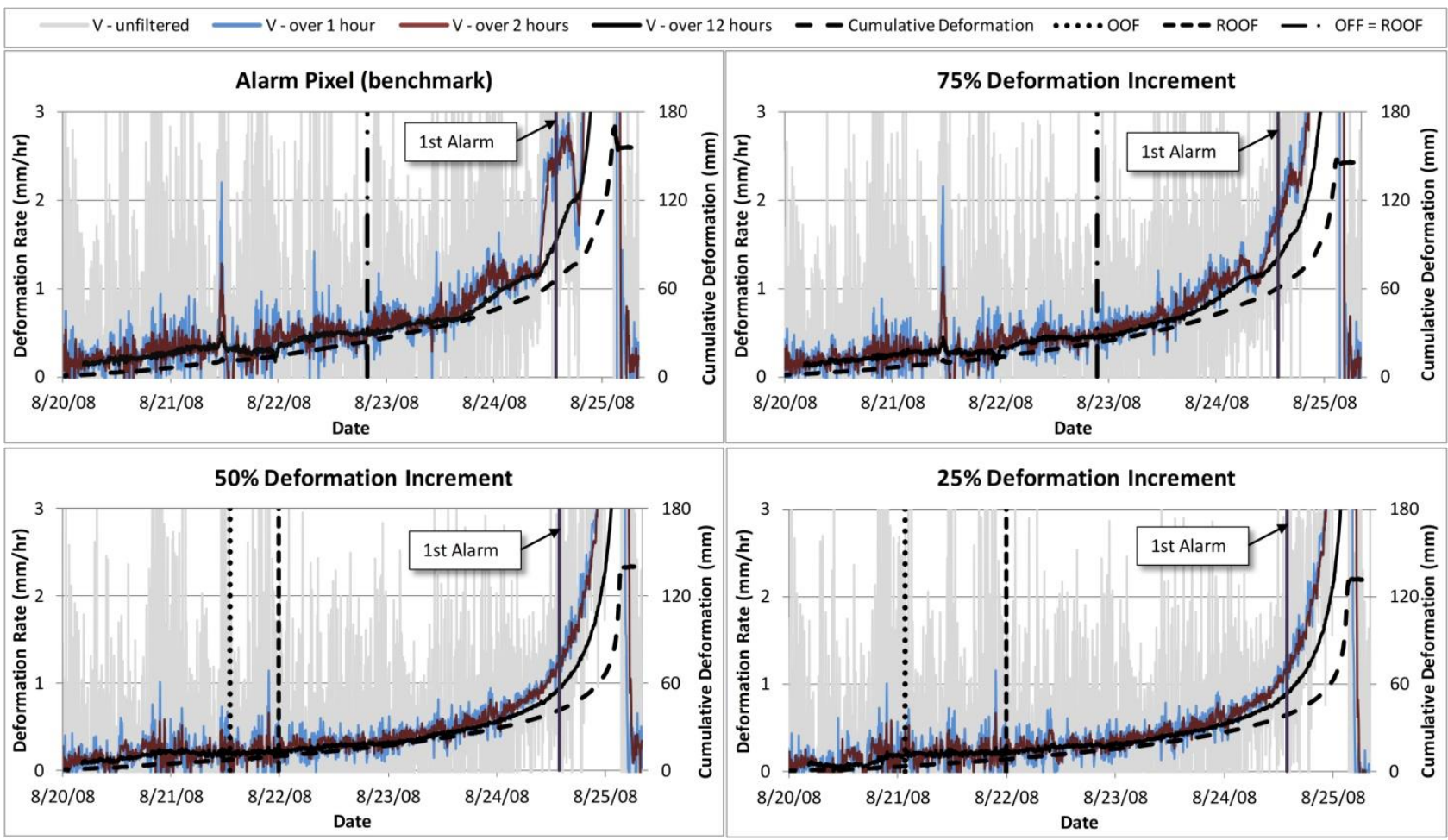

Figure 7 Cumulative deformation and deformation rate results for the copper mine slope failure using the spatial analysis method

\section{$4 \quad$ TOF prediction analysis}

The inverse-velocity and SLO methods were used to estimate the TOF for the copper mine slope failure using the spatial and temporal analysis method deformation rate results. The period of analysis for both TOF estimation methods is from the ROOF to rock mass collapse or failure (whichever comes first). Therefore, the TOF predictions are based on the selection of the ROOF. Data before the ROOF is not included in the TOF predictions. Data after the ROOF is cumulatively included providing an updated TOF prediction with each progressive scan. All four spatial analysis cases (benchmark and 75, 50 and 25\% benchmark deformation increments) were analysed and the results are presented in the following section.

\subsection{Inverse-velocity method TOF prediction results}

Figure 8 shows the inverse-velocity trends for the alarm pixel, and 75,50 , and $25 \%$ benchmark deformation increments from the estimated ROOF time to the slope failure point. The OOF and ROOF times are not the same for all cases, as discussed in Section 3, due to the averaging of multiple pixels.

TOF predictions were made by linearly extrapolating the inverse-velocity curve to the $x$-axis (inverse-velocity $=0$ ), as discussed in Section 2.1.1. The TOF was predicted for each data point beginning at the ROOF until the time of slope failure; this replicates the real-time updated TOF prediction based on 'new' data available after each radar scan using cumulative data from the ROOF point. The cumulative TOF predictions are best represented on 1:1 plots, comparing the actual and estimated TOF, termed 'life expectancy' plots. The life expectancy is defined as the difference between the predicted TOF and the time when the prediction is made. Figure 9 presents the life expectancy plots for each inverse-velocity trend analysed using the spatial and temporal method. Points with a positive life expectancy represent a prediction and a negative life expectancy indicates no prediction (predicted TOF is before the time the prediction was made). The 'actual life expectancy' line shown on the life expectancy plots represent the actual time from when a prediction is made to the time of slope collapse (not slope failure as it varies with each data set). Points above the actual life expectancy line indicate an over prediction of the actual time of slope collapse and the points below an under prediction. 


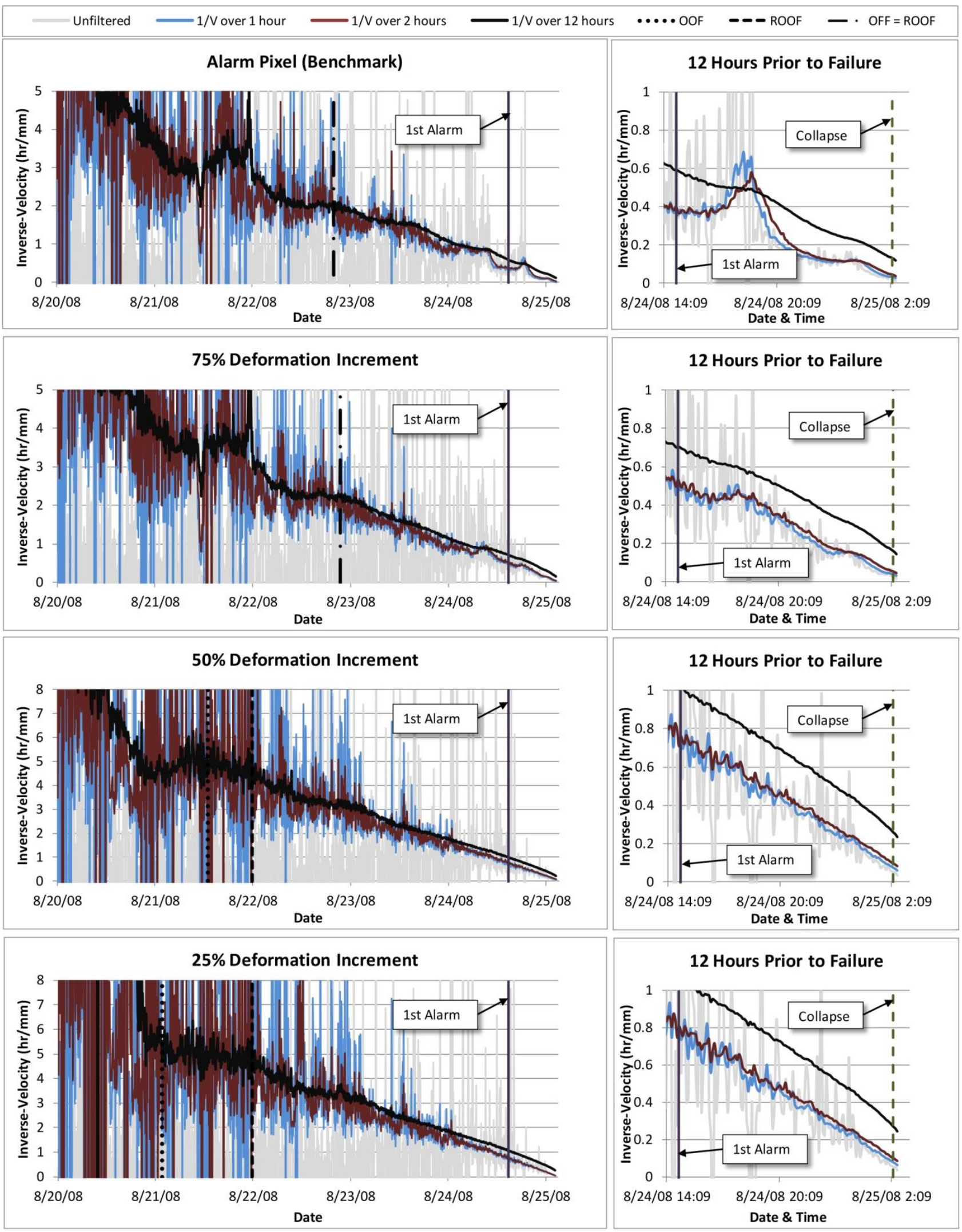

Figure 8 Copper mine slope failure inverse-velocity trends based on the spatial and temporal analysis method from SSR deployment to slope failure 

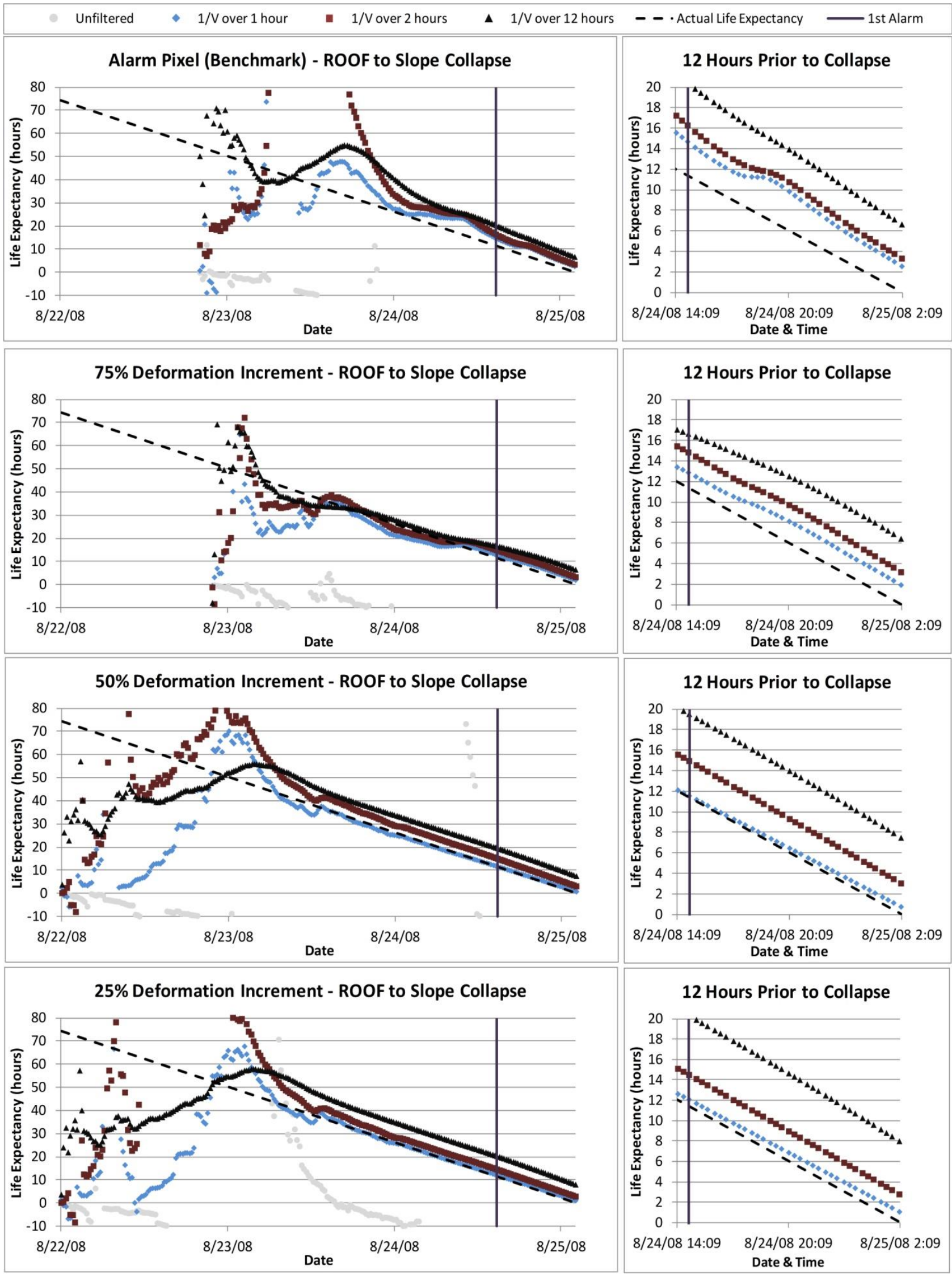

Figure 9 Copper mine slope failure life expectancy plots predicted using the inverse-velocity method based on the spatial and temporal analysis results from ROOF to slope collapse 


\subsection{SLO method TOF prediction results}

Figure 10 illustrates how the TOF can be estimated using the SLO method (50\% deformation increment results at the time of slope collapse presented as an example) by calculating the slope of the $t(d u / d t)$ versus $d u / d t$ line ( $T_{f p}$ in Figure 10), as discussed in Section 2.1.2. The $t(d u / d t)$ axis in Figure 10 represents the time multiplied by the deformation rate and does not represent the actual slope deformation.

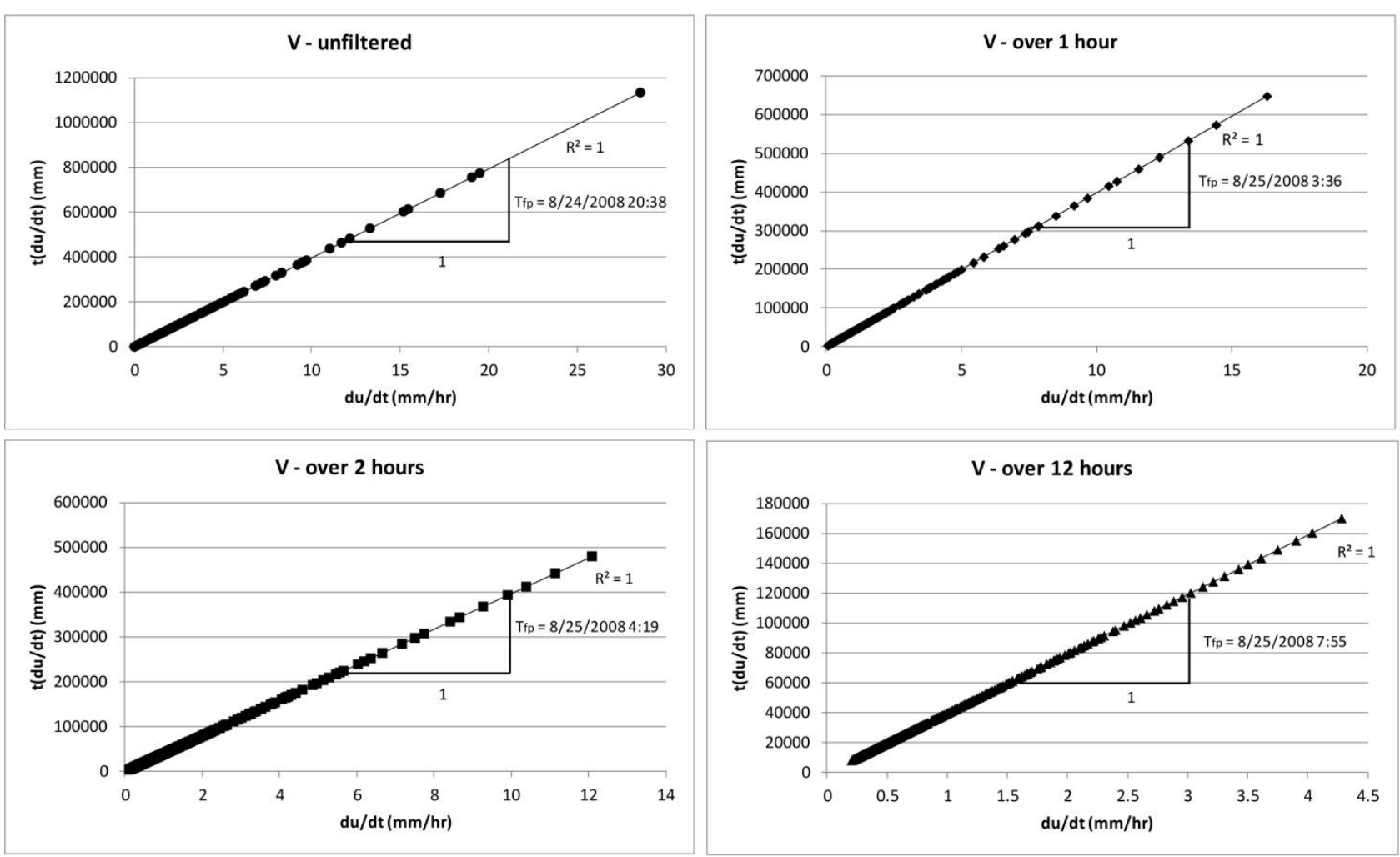

Figure 10 Copper mine slope failure SLO trends for $50 \%$ deformation increment from ROOF to slope collapse

Life expectancy plots are useful in illustrating TOF predictions for the SLO method. Similar to the inversevelocity life expectancy, the SLO TOF predictions can be made with each 'new' measurement from the radar scan by taking the slope of the cumulative $t(d u / d t)$ versus $d u / d t$ line beginning at the ROOF time. Figure 11 presents the life expectancy plots for each SLO trend analysed using the spatial and temporal method.

\section{$5 \quad$ Discussion of the results}

The main result of averaging the time-deformation over multiple pixels is the reduction in noise between progressive radar scan measurements. This decrease in noise becomes evident in the deformation and deformation rate versus time plots (Figure 7) where increasing pixel selection areas result in smoother deformation-time trends, and as a consequence smoother deformation rate versus time trends; especially when the deformation rate filtering technique is applied (see Equation 3).

The less noisy the deformation rate versus time data, the easier it is to apply the inverse-velocity and SLO TOF prediction methods. The life expectancy plots for the inverse-velocity (Figure 9) and SLO (Figure 11) methods show convergence with the actual life expectancy line earlier with increased amount of pixels included in the analysis. The use of life expectancy plots when making TOF predictions have shown to be very useful in illustrating TOF predictions in this case study. Life expectancy plots however are not very prevalent in case studies found in literature; it is recommended that they be considered in TOF prediction. 


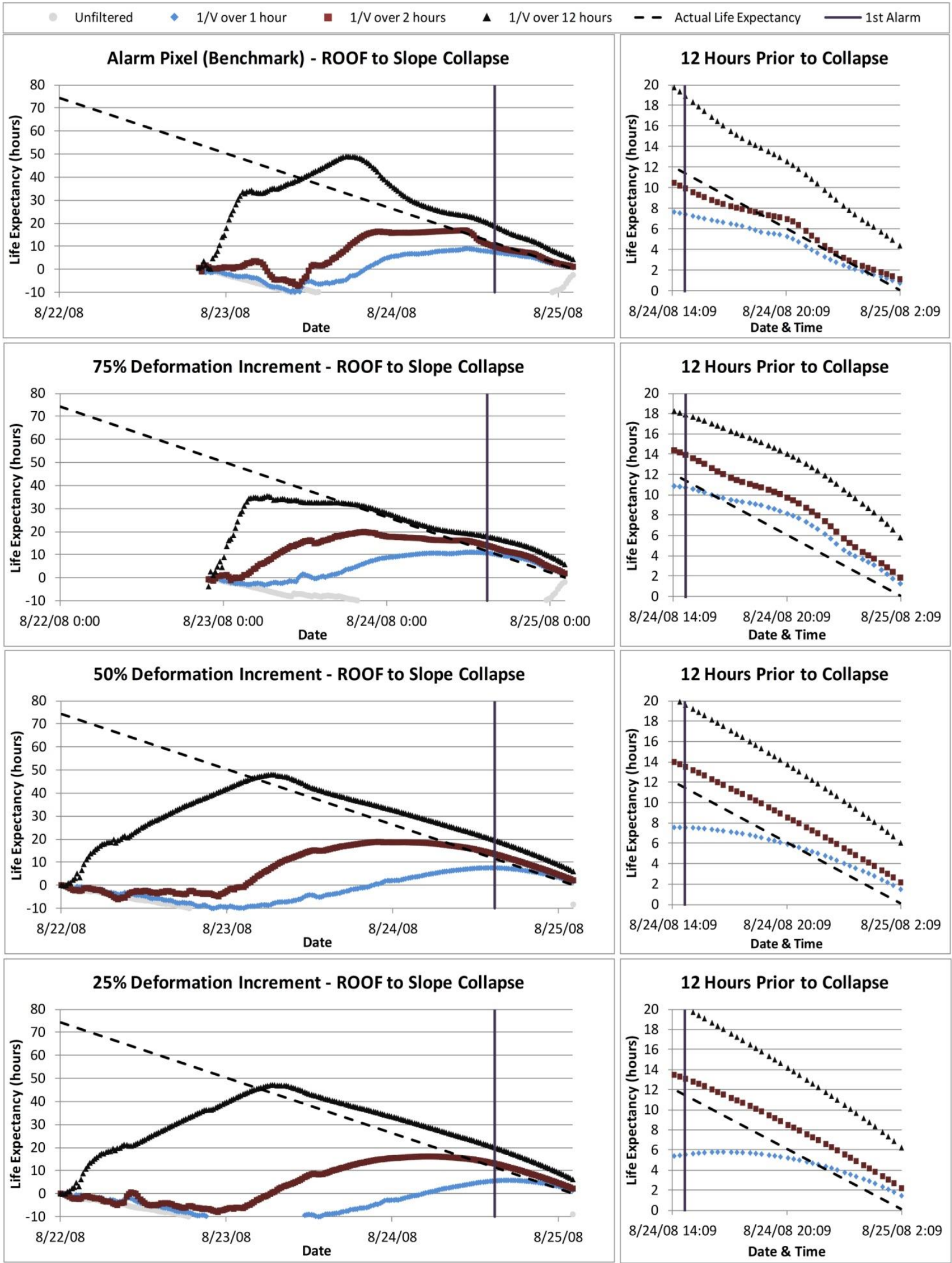

Figure 11 Copper mine slope failure life expectancy plots predicted using the SLO method based on the spatial and temporal analysis results from ROOF to slope collapse 
In the copper mine slope failure case study, the increased amount of pixel selection permitted an earlier estimation of the onset-of-failure (OOF) and revised onset-of-failure (ROOF) times, allowing the inverse-velocity and SLO TOF prediction methods to be applied sooner in the data set. For example, when compared to the single alarm pixel, the OOF was estimated to be over a day before using the $50 \%$ deformation increment, and up to two days earlier with the $25 \%$ deformation increment, depending on how much noise is filtered out of the data. Table 2 shows the time before slope collapse when an 'accurate' TOF prediction was made based on the life expectancy plots; taken where the TOF prediction converged linearly with the slope of the actual life expectancy line (N/P indicates no prediction). Using the inverse-velocity method in conjunction with deformation rate filtering (Equation 3 ), the time of slope collapse was predicted between 35 and 40 hours prior to collapse for the 50 and $25 \%$ deformation increments. This advanced recognition of the OOF time, and as a result the ROOF, is significant to early detection of impending slope failure. Overall, the SLO method did not give early accurate TOF predictions. The late SLO TOF predictions may be a consequence of starting the analysis too soon while there is still significant noise in the data. The SLO method may be better suited to making TOF predictions beginning later than the OOF time.

Table 2 Time before slope collapse where an 'accurate' TOF prediction was made

\begin{tabular}{ccccccccc}
\hline \multirow{2}{*}{$\begin{array}{c}\text { Deformation } \\
\text { Increment }\end{array}$} & \multicolumn{2}{c}{ V-Unfiltered } & \multicolumn{2}{c}{ V-Over $\mathbf{1}$ Hour } & \multicolumn{2}{c}{ V-Over $\mathbf{2}$ Hours } & \multicolumn{2}{c}{ V-Over 12 Hours } \\
& INV & SLO & INV & SLO & INV & SLO & INV & SLO \\
\hline Alarm* (hrs) & N/P & N/P & 15 & 6 & 15 & 12 & 20 & 15 \\
$\mathbf{7 5 \% ~ ( h r s ) ~}$ & N/P & N/P & 20 & 10 & 20 & 15 & 35 & 30 \\
$\mathbf{5 0 \% ~ ( h r s ) ~}$ & N/P & N/P & 35 & 6 & 35 & 15 & 40 & 40 \\
$\mathbf{2 5 \%}$ (hrs) & N/P & N/P & 35 & 5 & 35 & 15 & 40 & 40 \\
\hline
\end{tabular}

*Mines selected alarm threshold based on a single pixel.

In the copper mine slope failure case study, the $50 \%$ deformation increment combined with the filtered deformation rates over one and two hours seemed to yield the best TOF prediction results using the inverse-velocity method based on the earliest 'accurate' prediction of slope collapse. For the purpose of advanced detection, deformation rates filtered over 12 hours were useful in determining an early OOF time and predicting impending failure sooner. However, deformation rates filtered over 12 hours are not accurate for TOF predictions on the order of hours prior to slope collapse. Unfiltered deformation rates were not useful in early detection days prior to slope collapse but may be useful when predicting failure on the order of hours prior to collapse.

A limitation of this spatial and temporal analysis method is the heavy reliance on the determination of the OOF and ROOF times as the basis for the TOF predictions. The determination of the OOF and ROOF times through interpreting time versus deformation, deformation rate, and inverse-velocity curves can be subjective to the geotechnical engineer interpreting the results. Although selection of the OOF and ROOF times based on time versus deformation, deformation rate, and inverse-velocity curves is not unreasonable, a more systematic way of selection is being developed.

The large increase in analysis pixel area between the 75 and $50 \%$ deformation increments may be an indication that the copper mine slope deformation warning alarm threshold was set too high. Lower or multi-tiered thresholds across multiple pixels may have indicated the imminence of slope failure sooner. It is important to re-iterate that the benchmark and deformation increment percentages presented in this paper are only an example and can be selected by the user at their discretion. For example, the benchmark pixel can begin as the first alarm but can then be changed to a later alarm pixel when more radar measurements become available based on the judgement of the geotechnical engineer. 


\section{$6 \quad$ Concluding remarks}

A new methodology for spatial and temporal analysis of mine slopes using ground-based radar has been presented. The proposed spatial and temporal methodology is a versatile analysis tool designed to be used systematically in real time as slope alarms sound and new radar measurements become available. As an example, the methodology was applied to a slope failure at an open pit copper mine. The results were used to test the inverse-velocity and SLO time of failure (TOF) prediction methods which gave good results 35 to 40 hours prior to slope collapse; as compared to 15 hours for the single pixel threshold that was used to trigger an alarm prior to failure. Finally, the results presented indicate the importance of evaluating different monitoring threshold levels and varying number of pixels in TOF prediction analysis rather than relying on information from single pixels alone. This process should be implemented by the geotechnical engineer on an ongoing basis when evaluating slope movement trends and potential for slope failure.

\section{Acknowledgement}

The Authors thank Albert Cabrejo and David Noon at GroundProbe for their time and effort in providing SSR data sets for this research. Funding for this research was provided in part through a British Columbia Innovation Council Natural Resources and Applied Sciences (NRAS) Endowment grant.

\section{References}

Fukui, K. and Okubo, S. (1997) Life expectancy and tertiary creep for rock, in Proceedings of the Fall Meeting of Mining and Materials Processing Institute of Japan, Tokyo, Japan, Mining and Materials Processing Institute of Japan, Tokyo, pp. 91-94.

Fukuzono, T. (1985) A new method for predicting the failure time of a slope, in Proceedings Fourth International Conference and field workshop on landslides, Tokyo, Japan, Japan Landslide Society, Tokyo, pp. 145-150.

GroundProbe Pty Ltd (2012) SSRViewer 5.4 User Manual, GroundProbe Pty Ltd, Brisbane.

Harries, N., Noon, D. and Rowley, K. (2006) Case studies of slope stability radar used in open cut mines, Stability of Rock Slopes in Open Pit Mining and Civil Engineering Situations, Southern African Institute of Mining and Metallurgy, Johannesburg, pp. 335-342.

Mercer, K.G. (2006) Investigation into the time dependent deformation behaviour and failure mechanisms of unsupported rock slopes based on the interpretation of observed deformation behaviour, PhD Thesis, University of Witwatersrand, Johannesburg, South Africa.

Mufundirwa, A., Fujii, Y. and Kodama, J. (2010) A new practical method for prediction of geomechanical failure-time, International Journal of Rock Mechanics and Mining Science, Elsevier, pp. 1079-1090.

Reeves, B., Noon, D., Stickley, G. and Longstaff, D. (2001) Slope stability radar for monitoring mine walls, in Proceedings SPIE 4491 Subsurface and Surface Sensing Technologies and Applications III, 27 November 2001, San Diego, USA, International Society for Optics and Photonics, Bellingham, pp. 57-67.

Rose, N.D. and Hungr, O. (2007) Forecasting potential rock slope failure in open pit mines using the inverse velocity method, International Journal of Rock Mechanics and Mining Sciences, Vol. 44, Elsevier, pp. 308-320.

Zavodni, Z.M. and Broadbent, C.D. (1980) Slope failure kinematics, in Bulletin Canadian Institute of Mining, Canadian Institute of Mining and Metallurgy, Vol. 73, pp. 69-74. 